\title{
Effect of microstructure on fatigue crack tip field of laser melting deposited Ti-5Al-5Mo-5V-1Cr-1Fe titanium alloy
}

\author{
Kai Wang ${ }^{1}$, Rui Bao ${ }^{1, *}$, Siyuan Ren ${ }^{1}$, Dong Liu ${ }^{2,3,4}$ and Chuliang Yan ${ }^{1}$ \\ ${ }^{1}$ Institute of Solid Mechanics, Beihang University (BUAA), Beijing 100191, China \\ ${ }^{2}$ National Engineering Laboratory of Additive Manufacturing for Large Metallic Components (Beihang University), Beijing 100191, \\ China \\ ${ }^{3}$ Engineering Research Center of Ministry of Education on Laser Direct Manufacturing for Large Metallic Components, Beijing 100191, \\ China \\ ${ }^{4}$ School of Materials Science and Engineering, Beihang University, Beijing 100191, China
}

\begin{abstract}
In this paper, experimental and simulated investigations were carried out to understand the effect of microstructure on the fatigue crack tip strain field of laser melting deposited $\mathrm{Ti}-5 \mathrm{Al}-5 \mathrm{Mo}-5 \mathrm{~V}-1 \mathrm{Cr}-1 \mathrm{Fe}$ titanium alloy. The mechanical properties of the laser melting deposited material corresponding to different microstructure zones were measured by digital image correlation method under in-situ static stretching, and the results indicate that the direction of primary $\alpha$ lamellas has great influence on the mechanical property of each grain. The crack tip strain fields considering the influence of material microstructure under constant amplitude conditions were measured, and the strain field of plastic zone has significant difference. Crystal plasticity finite element simulation of the static tensile and crack tip field considering the microstructure effect had been conducted and compare with experiment results. The simulation results are in good agreement with the test results in both the static tension strain field and crack tip strain field.
\end{abstract}

\section{Introduction}

Crack tip field is very important to the investigation of fatigue crack growth behavior of various materials [12]. Traditional fracture mechanics, which is based on material homogeneity and isotropy hypothesis, has greatly developed and solved many engineering practical problems in many years. As a technique of additive manufacturing, laser melting deposition (LMD) is a rapid solidification material additive layered manufacturing technology for building components from a computer-aided design model [3-4]. The macrostructure morphology of LMD titanium alloy is featured by fine equiaxed grains and long columnar grains alternately arrayed due to the high temperature gradient in the overlap zone and relatively low temperature gradient in the body zone of the molten pool [5-7]. And the mechanical properties present inhomogeneity and anisotropy affected by the special microstructure [8-13], which brought great challenges to the research of the crack tip field.

There are amounts researches focused on the effect of microstructure on the fatigue crack growth (FCG) behavior of additive manufactured titanium alloys. Lu [14] observed a periodic fluctuation in the crack growth rate of LMD Ti-6.5Al-3.5Mo-1.5Zr-0.3Si alloy under constant amplitude loading, which was resulted from the layered structure with heat-affected bands. Acceleration and retardation in fatigue crack growth rates are observed during microstructurally-small fatigue crack propagation of laser engineered net shaped Ti-6Al-4V alloy due to the blocking effects at prior $\beta$ grain boundaries [15]. Xie et al. claimed that the FCG is dominated by the intrinsic crack resistance of the samples when $\Delta \mathrm{K} \geq \Delta \mathrm{K}_{\mathrm{T}}$ [16]. Galarraga et al. found that increasing the stress ratio can reduce the influence of microstructural features on region II crack growth in the electron beam melted Ti-6Al-4V ELI alloy [17]. However, few studies focus on the effect of special microstructure on fatigue crack tip field of additive manufactured titanium alloys.

In this paper, experimental and simulated investigations were carried out to understand the effect of microstructure on fatigue crack tip field of LMD Ti$5 \mathrm{Al}-5 \mathrm{Mo}-5 \mathrm{~V}-1 \mathrm{Cr}-1 \mathrm{Fe}$ titanium alloy. The mechanical properties of the material corresponding to different microstructure zones were measured by digital image correlation (DIC) method under in-situ static stretching. The crack tip field with the influence of microstructures were also measured and compared. Crystal plasticity finite element method (CPFEM) simulation of the static tensile and crack tip field considering the microstructure effect had been conducted and compare with experiment results.

\section{Experiment and simulation}

The Ti-5Al-5Mo-5V-1Cr-1Fe (Ti-55511) alloy, known as TC18 in China, is a typical near $\beta$ titanium alloy with

* Corresponding author: rbao@buaa.edu.cn 
high strength, high toughness and excellent fatigue property [18]. The specimens used in this study were cut from a $300 \mathrm{~mm} \times 100 \mathrm{~mm} \times 40 \mathrm{~mm}$ LMD TC18 thick plate. The macrostructure morphology of LMD TC18 titanium alloy is a typical equiaxed and columnar grains alternately arrayed, as shown in Fig. 1a. The microstructure inside the grains is characterized by elongated primary $\alpha\left(\alpha_{p}\right)$ lamellas orderly arranged, as shown in Fig. 1b. However, the directions of $\alpha_{p}$ lamellas in each grain are not same.

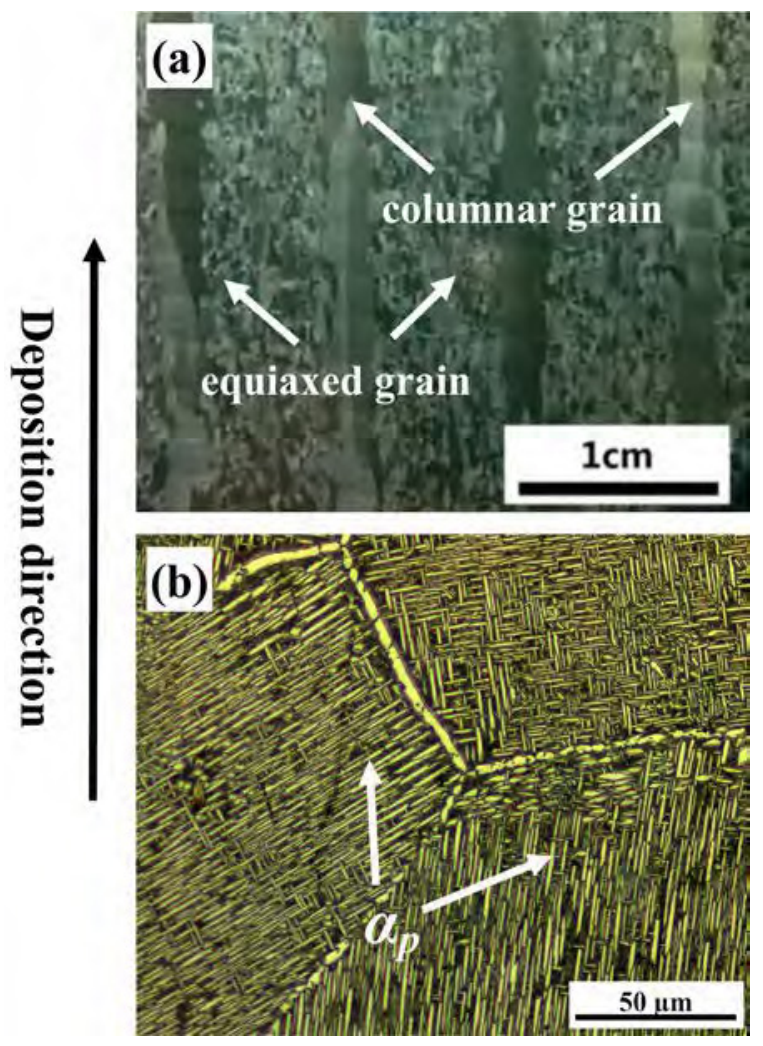

Fig. 1. The macrostructure (a) and microstructure (b) of LMD TC18 alloy.

Two kinds of specimens were used for the research: static tensile specimen to investigate the mechanical properties of various grains with different grain orientations, and single edge notched tensile (SENT) specimen to measure the crack tip strain field with different material characteristics. The nominal sizes of static tensile and SENT specimen used are shown in Fig. $2 a$ and Fig. $2 b$, and the thickness of both specimens is approximately $1 \mathrm{~mm}$. The notch direction of SENT specimen is parallel to the deposition direction. An insitu stretching observation system, mainly consisted of ZEISS AxioScope metalloscope, CARE Measurement \& Control Co IBTC-5000 in-situ tension testing machine and VIC-2D Digital Image Correlation software, as shown in Fig. 3, was used to measure the specimen deformation under static stretching and cyclic loading. Both surfaces of all specimens were sanded step by step with $300,600,800,1200,2000$ sandpapers and then etched by a mixture of $1 \mathrm{ml} \mathrm{HF}, 6 \mathrm{ml} \mathrm{HNO}_{3}$ and $100 \mathrm{ml}$ $\mathrm{H}_{2} \mathrm{O}$ to provide sufficient features as speckles for the DIC test and observe the metallographic structure meanwhile.
Static tensile test use a $100 \mathrm{X}$ total magnification with a field of view approximately $1.25 \times 1.0 \mathrm{~mm}$. Displacement step-by-step loading was used during the in-situ static tensile test until destroyed, and take three photos at each step to avoid random errors caused by factors such as vibration. As for the test of the crack tip strain field measurement, the crack of SENT specimens should firstly be prefabricated to $3.0 \mathrm{~mm}$ with a stress ratio 0.1 , and the last step maximum load of gradually lower alternating load is $80 \mathrm{MPa}$. Then use force loading with $0 \mathrm{MPa}, 0.1 \times 80 \mathrm{MPa}$ and $80 \mathrm{MPa}$ three steps, and also take three photos at each level of load.

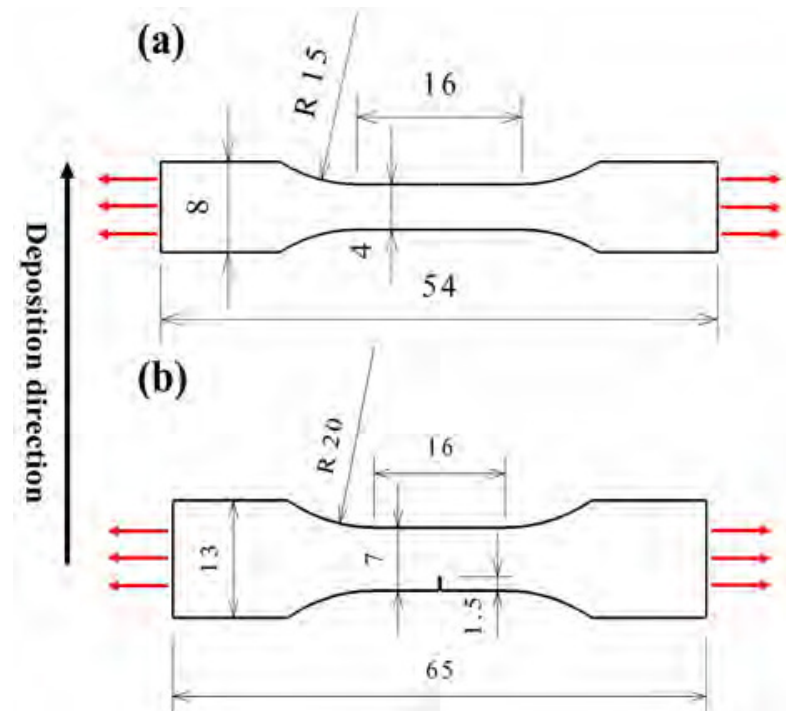

Fig. 2. Specimens nominal sizes: (a) static tensile specimen, (b) SENT specimen.

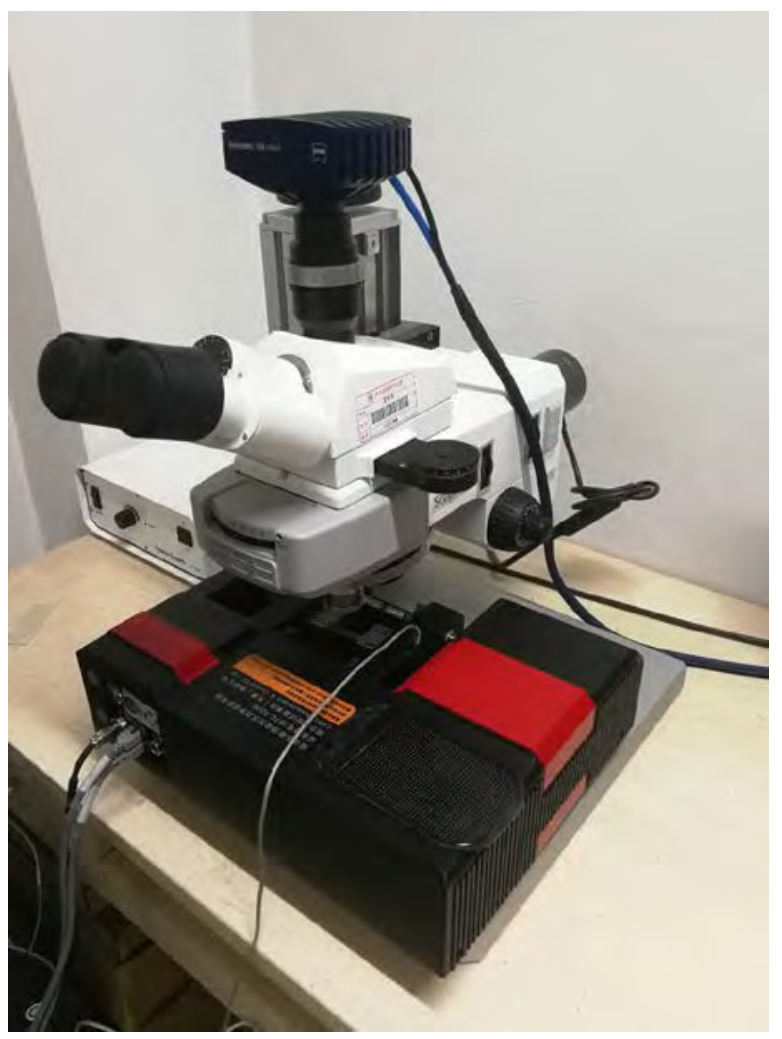

Fig. 3. In-situ stretching observation system 
Crystal plasticity finite element method (CPFEM) was used to calculate static tensile and crack tip field considering the microstructure effect. A threedimensional geometric model was established based on the Voronoi diagram principle as shown in Fig. 4a, and its seed points were optimized to make the grain size controllable. The orientation of each grain is random. The Python scripting language was used to build an optimized mesh model in ABAQUS, and a multi-step calculation was performed based on this model. The similar method was used to create a monocrystal model with crack as shown in Fig. 4b. Change the grain orientation in calculations to investigate its effect on the crack tip field. A force loading was used, and the loading direction has been marked in the figure.
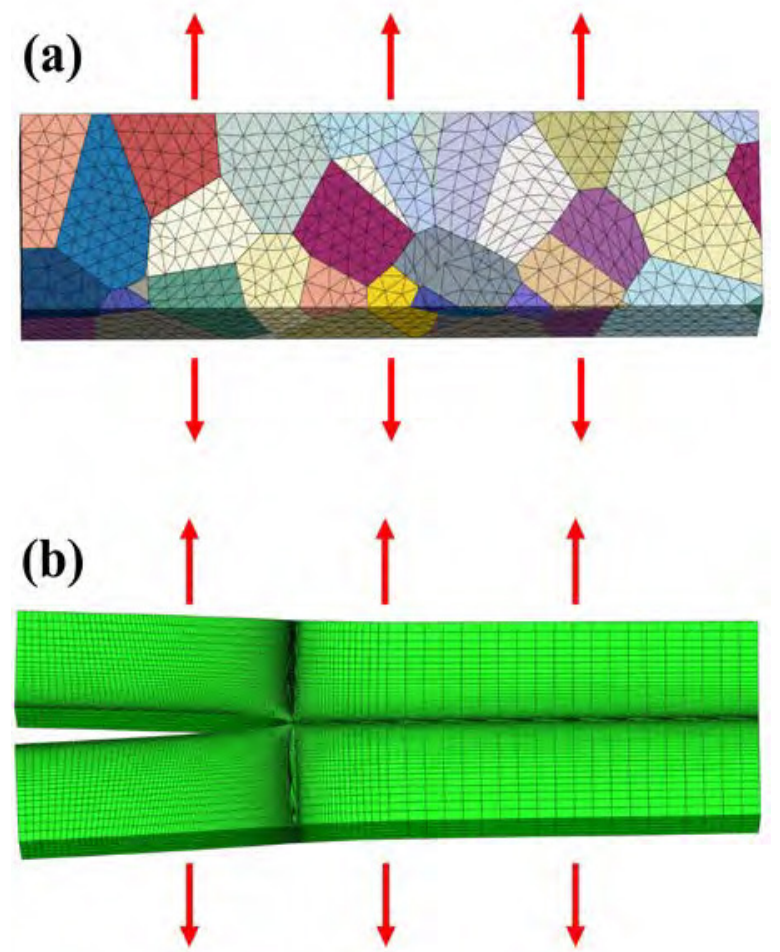

Fig. 4. CPFEM model: (a) static tensile polycrystalline model, (b) monocrystal model with crack.

\section{Results and discussion}

\subsection{In-situ static stretching}

The microstructure of measurement area is shown in Fig. $5 \mathrm{a}$, in which the direction of loading and $\alpha_{p}$ lamellas are also marked. The corresponding stretch direction strain field of displacement loading size $\Delta d i s=1.0 \mathrm{~mm}$ are shown in Fig. 5b. The results indicate that the great include angle of adjacent $\alpha_{p}$ lamellas direction would cause significant stress concentration at the grain boundary, as marked by circle in Fig. 5b, which is coincide with the effect of grain orientation.

In addition, the deformation of those grain with large angle $\alpha_{p}$ lamellas is observably lower, just as the purple area in Fig. $5 \mathrm{~b}$. The strain field of $\Delta d i s=0.4 \mathrm{~mm}$ shown in Fig. $5 \mathrm{c}$ gives a better illustration of this problem. If take
$0.2 \%$ as the yield standard, the whole specimen was already yield in $\Delta d i s=0.4 \mathrm{~mm}$. However, the tensile strain value of the area indicated by red is significantly greater than that of the purple area. It may due to the retardation of large angle $\alpha_{p}$ lamellas to the slip caused by maximum shear stress, the direction of which is $\pm 45^{\circ}$. At the same time, there are many deformation bands along $\pm 45^{\circ}$ in Fig. $5 \mathrm{~b}$ and Fig. $5 \mathrm{c}$, which should also be caused by the maximum shear stress.
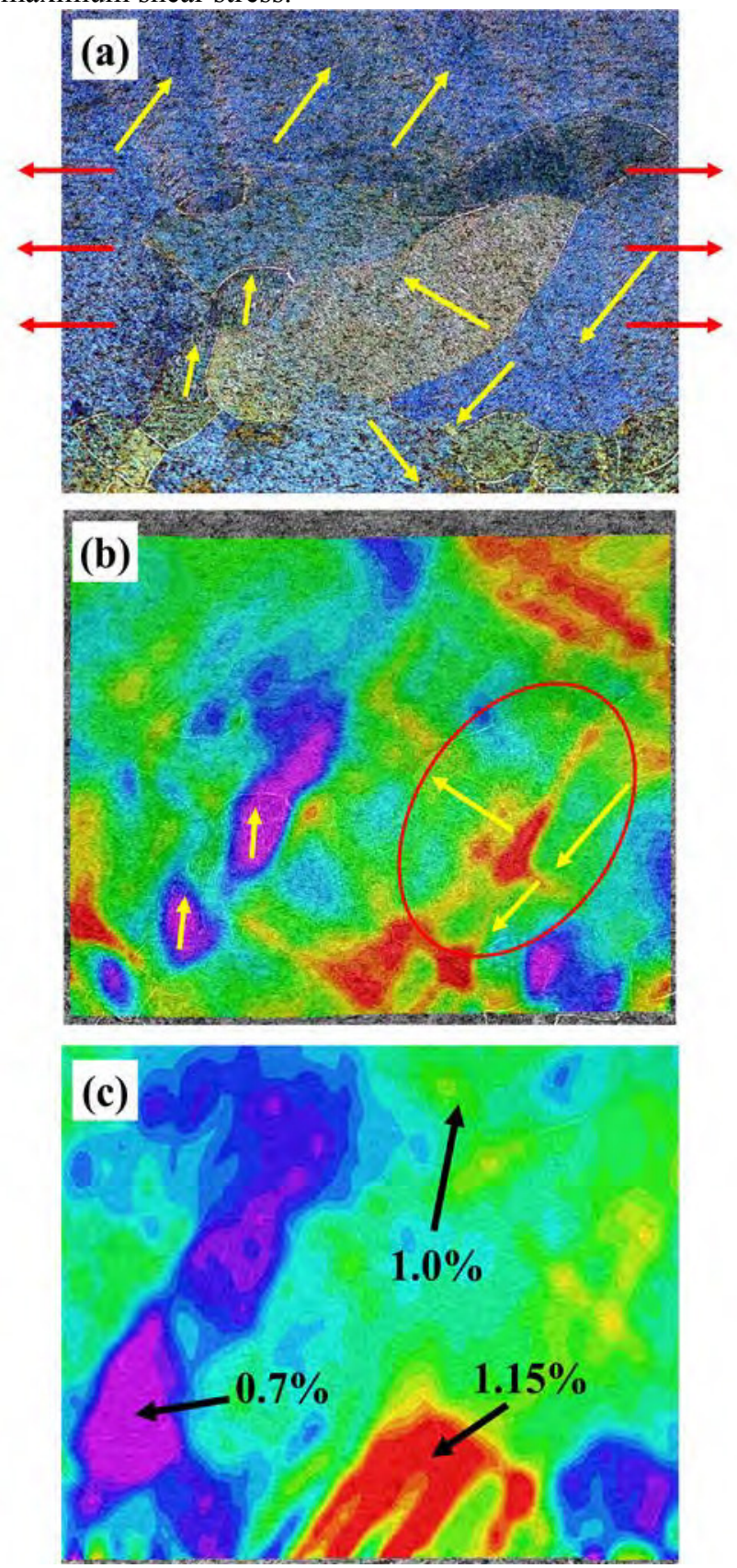

Fig. 5. (a) The microstructure of measure area; The strain field of stretch direction measured by DIC method: (b) displacement loading size $\Delta d i s=1.0 \mathrm{~mm}$, (c) $\Delta d i s=0.4 \mathrm{~mm}$.

Unlike plastic deformation, the elastic deformation of various microstructure is very close. In other words, the elastic property of different microstructure is similar. It may due to the totally different mechanism of elastic and plastic deformation. The elastic deformation of metal is caused by the length change of metal bond under normal 
stress. While the plastic deformation is achieved by dislocations and slips under shear stress. It also proves that the effect of $\alpha_{p}$ lamellas is mainly retard the dislocations and slips caused by shear stress.

The stretch direction strain field calculated by CPFEM is shown in Fig. 6. The results indicate that the deformation of those grains with random orientation is also different, which is similar with the experiment results.

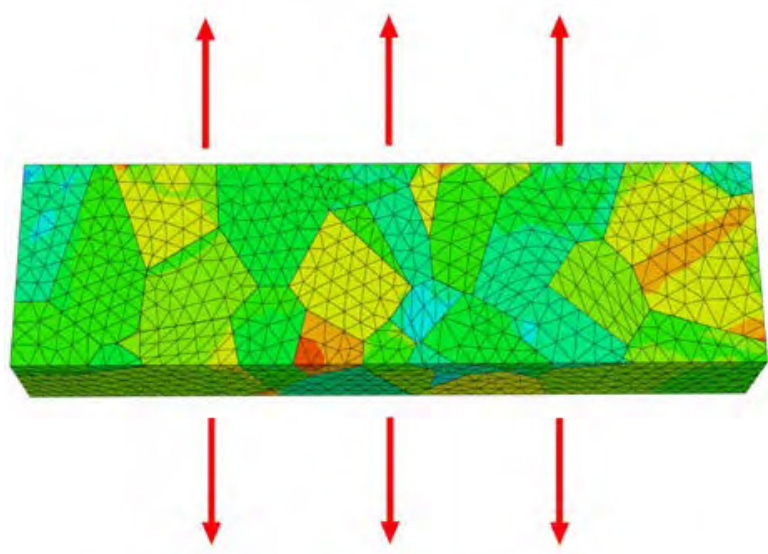

Fig. 6. The static tensile result of different grain orientation calculated by CPFEM.

\subsection{Crack tip strain field}

The crack tip strain fields measured by DIC method are shown in Fig. 7. The specimen was stretched along $X$ direction and the crack is along $\mathrm{Y}$, as the coordinate system shown in Fig. 7a. The strain field of $\mathrm{X}$ direction exx is shown in Fig. 7a, which is a typical butterfly shape. The Y direction strain eyy and shear strain exy are respectively shown in Fig. $7 \mathrm{~b}$ and Fig. 7c.

As introduced above, the crack of several specimens with various microstructure were prefabricated to $3.0 \mathrm{~mm}$ firstly. And three specimens with straight crack were chosen to investigate the effect of microstructure on the fatigue crack tip field. Considering the strain in $\mathrm{X}$ direction is much larger than $\mathrm{Y}$ direction, only exx was chosen to compare. A straight line from the crack tip in the $\mathrm{Y}$ direction was chosen to compare, which is parallel to the crack and is the axis of symmetry of the crack tip field.

The relationship of $\mathrm{X}$ direction strain exx and the distance to the crack tip $r$ along the line chosen above are shown in Fig.8. The three cracks with different microstructure in crack tip area measured by DIC method are shown in Fig. 8a. Obviously there is a significant difference in the plastic zone, which size is approximately $r_{0} \leqq 0.10 \mathrm{~mm}$, but small difference in elastic region. It may due to the difference in elastic property at different microstructure is small, but the plasticity property is likely to have significant difference. It is worth noting that the correct choice of crack tip position makes the exx of three cracks does not continue to increase significantly at the crack tip, but gradually converges to a certain value. It conforms to the inference of elastic-plastic fracture mechanics that the strain at the crack tip is limited, not infinity.
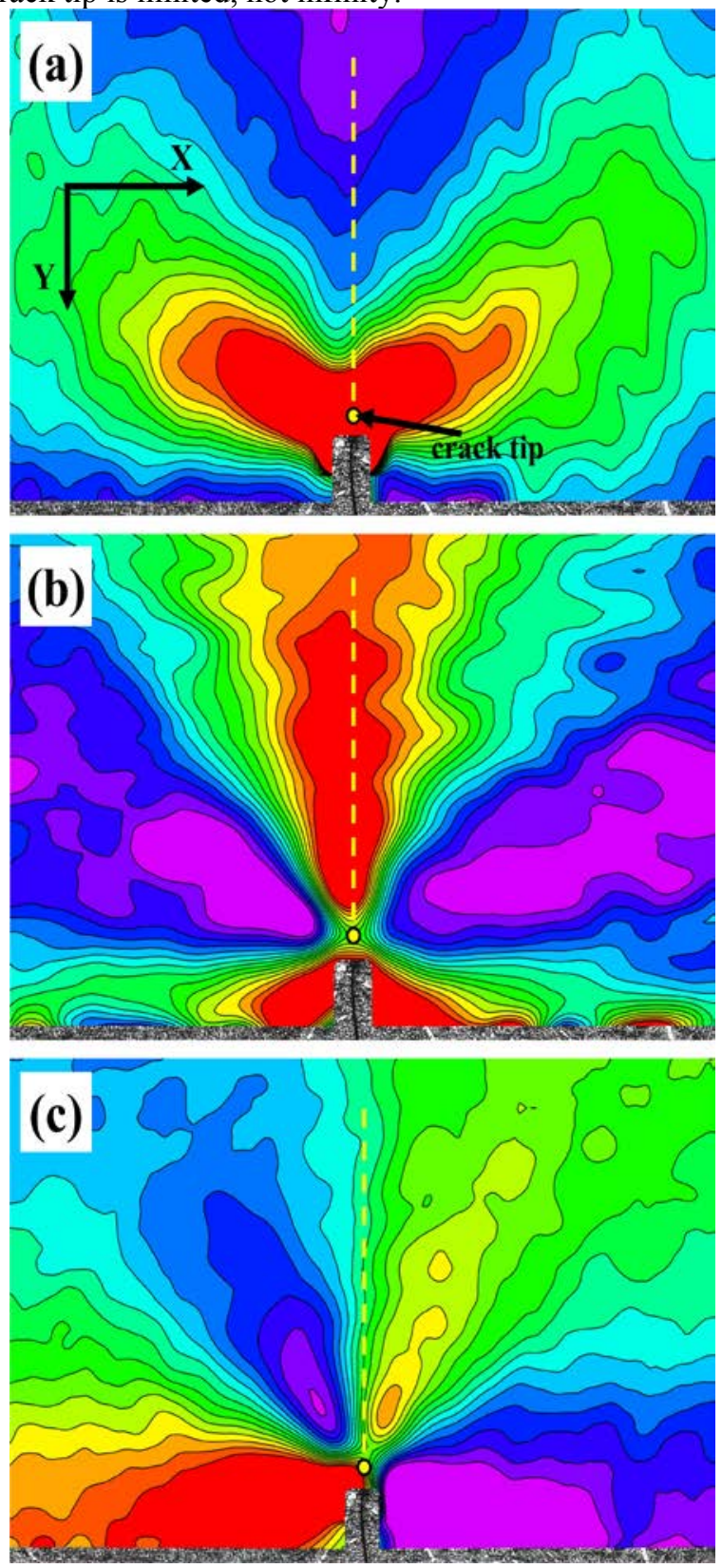

Fig. 7. The crack tip strain field measured by DIC method: (a) $\mathrm{X}$ direction (stretch direction) strain exx, (b) Y direction strain eyy, (c) shear strain exy 

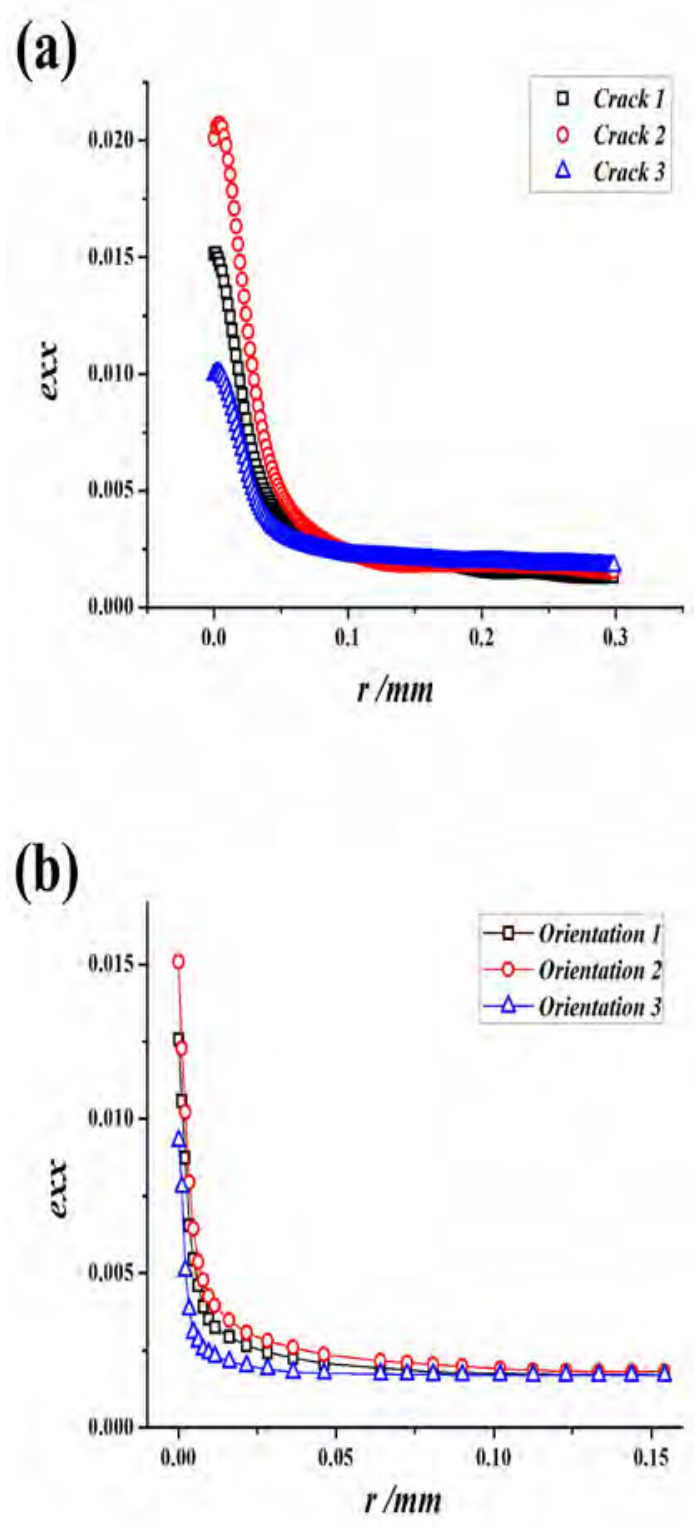

Fig. 8. The relationship of $X$ direction strain exx and the distance to the crack tip $r$ of: (a) three cracks with different microstructure in crack tip area measured by DIC method, (b) the same crack under different grain orientations calculated by CPFEM.

The results of three typical grain orientations calculated by CPFEM are shown in Fig. 8b. As the small size of the model, the load applied is smaller than test, thus the plastic zone is also smaller. However, the overall trend of exx with distance $r$ is similar to the results of experiments. More importantly, the difference between the three orientations in the plastic zone is significantly greater than that in the elastic zone, which is confirm to the conclusion of experiments.

\section{Conclusions}

In this study, the effect of microstructure on the fatigue crack tip field of laser melting deposited $\mathrm{Ti}-5 \mathrm{Al}-5 \mathrm{Mo}-$ $5 \mathrm{~V}-1 \mathrm{Cr}-1 \mathrm{Fe}$ titanium alloy was investigated. The main conclusions from this study can be summarized as follows:

1) The direction of orderly arranged $\alpha_{p}$ lamellas in each grain has significant effect on its mechanical property of LMD TC18 titanium alloy. The great include angle of adjacent $\alpha_{p}$ lamellas direction would cause significant stress concentration at the grain boundary. And the deformation of those grain with $\alpha_{p}$ lamellas direction deviates significantly from $\pm 45^{\circ}$ is observably lower.

2) The strain fields of plastic zone corresponding to cracks in different microstructure may have great difference, but the strain of elastic region is close. It may due to the difference in elastic property at different microstructure is small, but the plasticity property is likely to have significant difference.

3) CPFEM simulation results show good agreements with the test results on both static tension strain field and the crack tip strain field. The effect of the orientations of $\alpha_{p}$ lamellas on mechanical behaviors can be reflected by setting different grain orientations in CPFEM.

\section{Acknowledgement}

This work was supported by the National Natural Science Foundation of China (NO. 11672012)

\section{References}

1. J. M. Vasco-Olmo, F. A. Díaz, Eng. Fract. Mech. 166, 82-96 (2016)

2. S. E. Ferreira, M. A. Meggiolaro, Int. J. Fatigue (2018)

3. H. P. Qu, H. M. Wang, Mater. Sci. Eng. A, 466, 12 (2007)

4. G. K. Lewis, E. Schlienger, Mater. Des. 21, 4 (2000)

5. C. M. Liu, X. J. Tian, H. B. Tang, H. M. Wang, J. Alloys Compd. 572, 17-24 (2013)

6. Y. Zhu, D. Liu, X. Tian, H. Tang, H. Wang, Mater. Des, 567 (2014)

7. G. C. Li, J. Li, X. J. Tian, X. Cheng, B. He, H. M. Wang, Mater. Sci. Eng. A, 684, 233-238 (2017)

8. J. Alcisto, A. Enriquez, H. Garcia, S. Hinkson, T. Steelman, E. Silverman, et al. J. Mater. Eng. Perform. 20, 2 (2011)

9. H. Galarraga, Lados, R. R. Dehoff, M. M. Kirka, P. Nandwana, Addit. Manuf. 10, 47 (2016)

10. D. Agius, K. Kourousis, C. Wallbrink, T. Song,. Mater. Sci. Eng. A 701 (2017)

11. N. Shamsaei, A. Yadollahi, L. Bian, S. M. Thompson, Addit. Manuf. 8, 12-35 (2015).

12. X. Shi, S. Ma, C. Liu, Q. Wu, J. Lu, Y. Liu, et al. Mater. Sci. Eng. A, 684, 196-204. (2017) 
13. Q. Chunlei, J.E. Nicholas, Adkins, M. Moataz Attallah, Mater. Sci. Eng. A, 5788 (2013)

14. S. Lu, R. Bao, K.Wang, D. Liu, Y. Wu, B. Fei, Mater. Sci. Eng. A, 690, 378-386 (2017)

15. Y. Zhai, D. A. Lados, E. J. Brown, G. N. Vigilante, Int. J. Fatigue, 93, 51-63 (2016)

16. Y. Xie, M. Gao, F. Wang, C. Zhang, K. Hao, H. Wang, et al, Mater. Sci. Eng. A, 709 (2017)

17. H. Galarraga, R. J. Warren, D. A. Lados, R. R. Dehoff, M. M. Kirka, Eng. Fract. Mech. 176 (2017)

18. C. M. Liu, H. M. Wang, X. J. Tian, H. B. Tang, D. Liu. Mater. Sci. Eng. A, 586, 323-329 (2013) 